\title{
Application of Digital Image Cross Correlation to Study Sinkhole Collapse
}

\author{
Mahmoud Ahmed \\ Department of Transportation, Route 9A Project, Suite 1701, 115 Broadway, New York, NY 10006, USA \\ Correspondence should be addressed to Mahmoud Ahmed; mahmoud.ahmed@dot.ny.gov
}

Received 17 April 2013; Accepted 8 July 2013

Academic Editors: J. A. Entry, D. Hui, D. Lin, and W. R. Roy

Copyright ( 2013 Mahmoud Ahmed. This is an open access article distributed under the Creative Commons Attribution License, which permits unrestricted use, distribution, and reproduction in any medium, provided the original work is properly cited.

\begin{abstract}
This paper presents the results of a study using a transparent soil experimental technique and numerical modeling to detect 3D deformations resulting from submerged cavities that lead to a sinkhole. Excessive deformations from underground activity beneath highway pavements could lead to sinkhole collapse. The formation of a sinkhole is often sudden and can lead to extensive damage and loss of life, especially in urban areas. The use of transparent soils permitted the visualization of internal ground deformations which allowed for comprehensive evaluation of the extension of failure. A series of finite element analyses have also been carried out for the tests conditions. The observed sinkhole, at the surface, is found to be a small indicator of the final size and magnitude of the internal deformations as a subsequent funnel-shaped depression developed with a hole at the center. The modeling results emphasized the need to extend the repair zone following sinkhole collapse by a minimum distance that equals twice the cavity diameter away and ahead of the developed hole. Results of this study are believed to be of practical interest for predicting surface and internal ground deformations following sinkhole collapse which could be useful for the stability assessment of underground utilities and the development of a restoration plan after collapse occurred. The results also provided approximate bounds to areas affected by the sinkhole allowing for collapse risk to be assessed.
\end{abstract}

\section{Introduction}

Sinkholes are depressions that develop at the soil surface due to stress release in the soil beneath. They could develop naturally like in karst terrains, due to dissolution of limestone and the subsequent subsidence above the depression, or as a result of human underground activities such as horizontal directional drilling (HDD) or tunneling operation. They could also be formed due to leaking sewer or culvert by washing away surrounding soils. Risk of sinkhole collapse will continue to increase due to increased urbanization which intensifies the need to utilize underground space to accommodate large utility systems, subways, and highways, thereby decreasing traffic congestion and allowing preservation of aboveground space.

A sinkhole may develop on a roadway when an opening is formed, either beneath or within the subgrade layer, followed by the flowing of layers above into this opening. Such sinkholes require immediate and costly repairs to avoid further traffic delays and closures. The current maintenance approach to this problem is to quickly remove and/or repave damaged pavement. This approach may temporarily fix the surface problem. However, this may accelerate the underlying problem by increasing loads above the sinkholes and does not properly fix the foundation soils. Additionally, the problem is only fixed in areas that have currently failed, but nearby areas may still be on the verge of failure. If these pending failures can be detected, a more extensive and economical repair solution may be obtained and implemented. Nowadays, most of transportation agencies would not permit HDD or tunneling operation without proper assessment of the opening stability. Therefore, prediction of sinkhole collapse is important to insure the safe construction and protection of nearby utilities. However, the extension of the developed sinkhole and the occurrence of soil flow into the opening is complex phenomena and have yet to be well-understood. The relationship between ground movement, soil properties, and geometry of the opening is neither simple nor linear which made it difficult to achieve a comprehensive theoretical solution. Majority of previous studies on the subject 
of sinkhole formation are empirical in nature and usually associated with a specific ground condition. Other types of studies include analytical solutions, laboratory scale models, and numerical models. Major limitation in some of these methods widely used is that they neglect the possible soilstructure interactions. The objective of this research is to predict 3D deformations for a sinkhole collapse and develop a relationship between the geometry of the cavity and final deformations.

Sinkhole collapse is generally an instantaneous event which makes it difficult to get field measurement during the collapsing processes. Therefore, small-scale or full-scale experimental modeling may be a good practice to predict the size and shape of the sinkhole and to collect information about influential factors and triggering mechanism of a specific collapsing event. Full-scale experiments are expensive and difficult. Small-scale experiments have been reported by some authors such as Craig [1] and Abdulla and Goodings [2]. These authors focused on the stability of soils over cavities. Atkinson and Potts [3], Davis et al. [4], Mühlhaus [5], and Leca and Dormieux [6] used the underground cavity conditions in centrifuge testing to demonstrate the way in which soil around a circular cavity deforms as the overburden pressure increased and/or in situ stress released. The ultimate goal of these authors was to study the stability of lined and unlined tunnels by using circular cavity deformations. A similar approach has been adopted in this study by using transparent soil modeling technology and associated image processing techniques to investigate the aspects of sinkhole formation. A cylindrical cavity was preplaced inside a transparent soil model, which represents sand. The face of the cylinder was simulated using an internal pressure $\left(\sigma_{T}\right)$ equal to the effective stress at the center of the cavity and applied inside the cylinder. Tests were conducted by reducing $\sigma_{T}$ in stages until collapse of the soil occurred when the transparent soil caved in or flowed inside the cavity. The transparent soil permits the use of simple optical techniques to visualize $3 \mathrm{D}$ deformation patterns within the soil mass by slicing the model using a laser light sheet. In this study, the model was sliced parallel to and perpendicular to the cylinder axis in order to obtain 3D deformation patterns. Images of the soil, illuminated by a laser light sheet, were captured after each decrement of $\sigma_{T}$ and used to obtain the corresponding deformation fields at the precollapse and collapse stages. The technique might not model the exact construction sequencing of HDD or failure due to leaking sewer. Nevertheless, it demonstrates the way in which soil around a circular cavity deforms due to in-situ stress release which ultimately leads to sinkhole collapse. Transparent soil modeling has been used by the author before to study ground movements associated with tunneling [7] and tunnel face stability [8].

\section{Development of Transparent Soil Model}

Sinkhole failures usually result from reduction of in-situ stress at the face of excavated subsurface cavity. This process can be experimentally simulated by a contracting cylindrical cavity from the in-situ stress state to collapse. A simplified

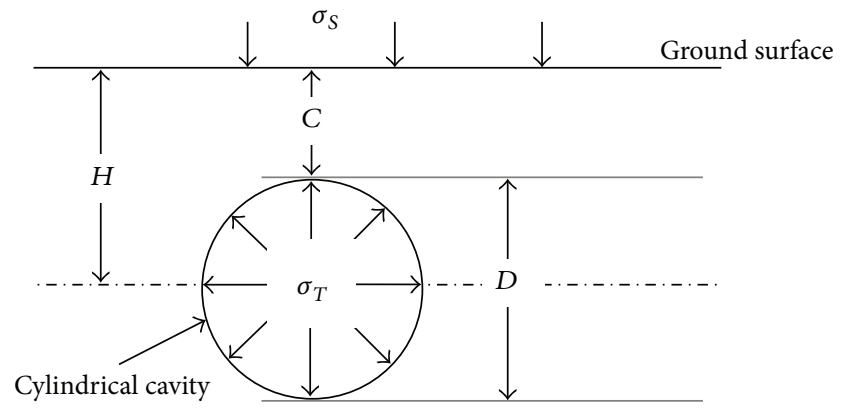

FIGURE 1: Simplified layout of collapsing cavity problem.

layout of the problem implemented in this study is shown in Figure 1. The submerged cavity is assumed to be cylindrical of diameter $D$ and cover $C$. The cavity was subject to an internal normal stress represented by internal pressure $\sigma_{T}$. The ground surface was horizontal and subject to a vertical surcharge $\sigma_{S}$. Plane strain was assumed.

2.1. Transparent Synthetic Soil. The transparent soil was made by matching the refractive indices of silica gel and pore fluids. For the preparation of the models, silica gel was immersed in the pore fluid. At the same time, the mix was stirred to release gas bubbles entrapped during the pouring of silica gel. Saturation of the particles was accomplished by soaking and mixing the particles in pore fluid under vacuum for several hours. Vacuum was applied to deair the mix until the mixture turned to transparent. Time for vacuum depends on the depth of silica gel and the power of the vacuum pump. Approximately 24 hours was required to fully saturate the silica gel. One advantage of transparent soil is that saturation can be readily viewed when the particles become transparent. The clarity of transparent soil depends on the perfect matching of the refractive indices of silica and pore fluid which was made by a blend of Drakeol35 mineral oil and Norpar 12 paraffinic solvent. The blend is $1: 1$ by weight. The refractive index, viscosity, and density of the oil blend at room temperature $\left(24^{\circ} \mathrm{C}\right)$ were $1.447,5.0 \mathrm{cp}$, and $800 \mathrm{~kg} / \mathrm{m}^{3}$, respectively. This mix resulted in a material which was completely transparent, thereby permitting the use of simple optical techniques to visualize $3 \mathrm{D}$ deformation patterns within the soil mass. Silica gel materials used in this study are produced by Multisorb Technologies located in Buffalo, New York, with particle size $0.5-1.5 \mathrm{~mm}$. The specific gravity of silica gel is 2.2 [9], which is approximately $80 \%$ of the specific gravity of natural silicate sands. The minimum density of silica gel determined in accordance to the American Society for Testing and Materials (ASTM) standard D4254 was $\gamma_{\min }=7 \mathrm{kN} / \mathrm{m}^{3}$. Because of the porous structure of the silica gel, ASTM standard D698 was not applicable. A maximum density $\gamma_{\max }=8 \mathrm{kN} / \mathrm{m}^{3}$ was obtained using a vibrator compaction. The saturated unit weight depends on the pore fluid used, and it was 11$14 \mathrm{kN} / \mathrm{m}^{3}$, for the mineral oil and solvent pore fluid used in this research. The volume change of the silica was consistent with that of natural sands. The hydraulic conductivity ranged from $1 * 10^{-2}$ to $1 * 10^{-4} \mathrm{~cm} / \mathrm{sec}(12-45$ Darcys), depending 


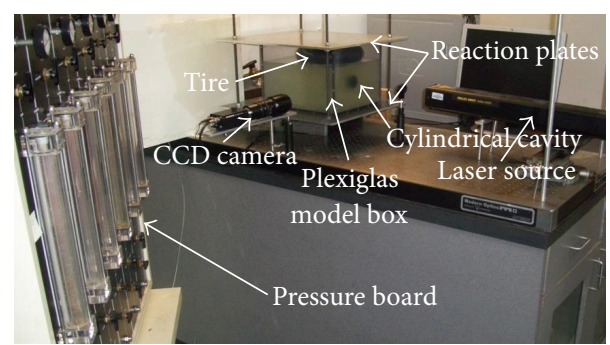

FIGURE 2: Test setup.

on grain size. The angel of friction is $30^{\circ}-36^{\circ}$, and Young's modulus is $24-84 \mathrm{MPa}$ depending on density and size. These values were similar to the values reported for the natural sands. The average stress strain behavior of silica gel is consistent with typical stress strain behavior of sand for both dense and loose conditions [10].

2.2. Experimental Modeling Technique. A plexiglas model (Figure 2) $30.50 \mathrm{~cm}$ long, $25.40 \mathrm{~cm}$ wide, and $20.30 \mathrm{~cm}$ high was used to contain the transparent soil. The model dimensions have been chosen based on parametric analysis (cavity size and depth), performed by empirical formulae, in such a way that the influence of the boundaries was minimized. Transparent soil was placed into the plexiglas model box at approximately $25 \mathrm{~mm}$ lifts. The box was shaken by hand while being packed to a density $\gamma=7.5 \mathrm{kN} / \mathrm{m}^{3}$. The contracting cylindrical cavity was modeled by a PVC tube of $2.50 \mathrm{~cm}$ diameter and $25.40 \mathrm{~cm}$ long preinstalled inside the model. A latex membrane $(0.3 \mathrm{~mm}$ thick $)$, of negligible strength, was attached to the end of the cylinder to represent the contracting cavity. The membrane was left slack to prevent mechanical influence on the displacement of the face. The cylinder was then filled with water under pressure $\left(\sigma_{T}\right)$ which can be read and controlled by a pressure board. A surcharge was needed to counter the effects of the small size model and the low-unit weight of the transparent soil. The surcharge may affect the scaling of stress-based problems such as scaling of liner deformations or soil reinforcement, to natural soils. For the deformational problems such as the one presented here, it is believed that the role of geometry is more significant than surcharge, but small differences in failure geometry may occur due to the presence of surcharge. For application of surcharge or surface pressure $\sigma_{S}$, the plexiglas model container was placed between two identical metal plates (Figure 2) connected by four threaded rods. A rubber tire with internal pressure $\sigma_{S}$ was placed on top of the transparent soil and connected to the pressure board. The cylinder pressure $\sigma_{T}$ was increased such that $\sigma_{T}=\sigma_{S}$ at the beginning of the test. In addition to the tunnel container, the setup also included a Cohu 2622 black and white Charged Coupled Device (CCD) camera, $35 \mathrm{~mW}$ Melles Griot laser light source, a line generator lens, a loading frame, a test table, and a PC for image processing (Figure 2). The camera has a resolution of $640 \times 480$ pixels and is controlled by the PC through a Matrox Meteor 2/4 frame grabber. A macrozoom lens with a variable focus length from 18 to $108 \mathrm{~mm}$ was mounted on the CCD camera.
The tests were conducted by reducing the cylinder pressure $\sigma_{T}$ in stages until collapse occurred. At some point, the cavity becomes sufficiently large and the remaining overburden is no longer able to arch across the cavity and collapsed. This process was found to be similar to in-situ cavity collapsing event. After each decrement of pressure, the model was sliced optically using laser light sheet to illuminate plane of measurements inside the model and an image was taken by CDD camera. Later, these images were processed to obtain corresponding deformations relative to pressure drop and volume loss in the soil mass induced by the cavity contraction. Complete strain and deformation fields were obtained from the set of images taken during the test.

\subsection{Deformation Measurement Using Digital Image Process-} ing. The measurement technique described in this paper operates by processing digital images, which can be captured directly from digital camera. The interaction between laser light and transparent soil produces a distinctive specklepattern. This speckle pattern manifests the interaction between the transparent soil matrix, impurities, entrapped air, and the laser. Small particle movement will result in a change in the speckle distribution in the plane of measurement. If the deformation is small, the contrast distribution resulting from the speckle effect will follow the particle movement. Images captured before and after deformations are analyzed using digital image correlation (DIC). The captured images are divided into a large number of interrogation areas or windows. It is then possible to calculate a displacement vector for each window with the help of DIC techniques. DIC has proven to be a valuable flow and deformation measurement tool that provides enhanced measurement capabilities not possible with other techniques and has been widely used for experimental measurements in solid mechanics and in fluid mechanics [11-13].

An advanced form of DIC which employs window shifting and window sizing, called adaptive cross correlation (ACC) has been adapted for this study. Comparison between conventional DIC and ACC demonstrated that ACC gives a better performance than conventional DIC. ACC not only reduces the prediction errors but also relieves the limit of the maximum reliable displacement, which depends on the interrogation window size. ACC uses both variable window sizing and window shifting. ACC permitted measuring deformation with a resolution of 0.1 pixels, which corresponded to a displacement on the order of $0.01 \mathrm{~mm}$ based on the CCD camera and the optical settings in the test. This accuracy can be improved by increasing the resolution of the camera or adjusting the optical setting, for example, using a smaller focus area. ACC is implemented in Flow Manager Software [14], which is the software used in this research.

\section{Finite Element Modeling}

PLAXIS 2D (V.8) has been used in this study. Soil properties, cavity size, and depth were chosen similar to the transparent soil models conditions. The pore pressure distribution was assumed to be hydrostatic and phreatic level was located at 


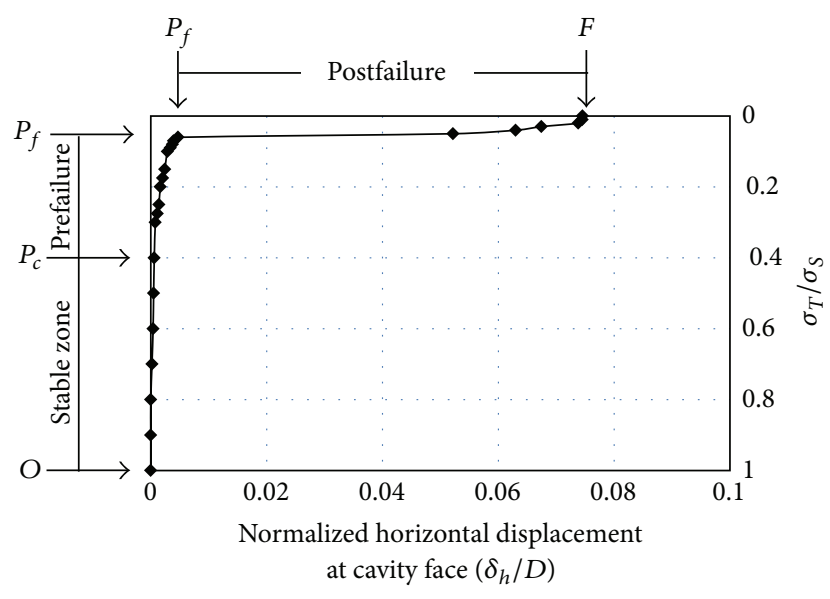

FIGURE 3: Plot of incremental cylindrical cavity contraction versus stress release.

the surface. The cylinder is modeled by curve plates with PVC properties and curved interface. All models were analyzed by assuming drained behavior for transparent soil, rigid interface strength, and elastic-plastic Mohr-Coulomb model. The input parameters used in PLAXIS were back-calculated from drained triaxial tests performed on transparent soil except the dilatancy angle which was assumed (unit weight $=9 \mathrm{kN} / \mathrm{m}^{3}$; cohesion $=0$, permeability $=1.5 * 10^{-4} \mathrm{~cm} / \mathrm{sec}$, Young's modulus $=21,000 \mathrm{kN} / \mathrm{m}^{2}$, Poisson's ratio $=0.45$, friction angle $=36^{\circ}$, and dilatancy angle $=1^{\circ}$ ). Staged construction with plastic calculation option was used to simulate the contracting cavity. The first stage included activation of the cylinder, deactivating of soil inside the cavity, and generation of the pore water pressure. The cavity development was simulated in the following stages by applying contraction measured from transparent soil models to the cylinder.

\section{Results and Discussion}

Multiple tests were performed at the same soil density for a single cavity size located at various depths. For a constant cavity size, the depth was found to be of little or no effect on final deformations. Therefore, only one set of results has been presented in this paper.

4.1. Deformations Due to Stress Release. Plot of incremental cylindrical cavity contraction versus stress release is presented in Figure 3. The stress release, represented by reduced support pressure $\sigma_{T}$ normalized by the vertical effective stress $\sigma_{V}$ at the cavity axis, is plotted against horizontal displacement $\delta_{h}$, is measured at the center of the cavity, and normalized by cavity diameter, $D$. As $\sigma_{T}$ is reduced, no movement has observed down to pressures that equal is $50 \%$ of the initial support pressure. Further reduction in support pressure, beyond point $P_{c}$ down to point $P_{f}$, resulted in small displacements, which are approximately proportional to the reduction in $\sigma_{T}$. Further reduction in supporting pressure results in a sudden collapse where larger deformations were measured without any further reduction in the supporting pressure.

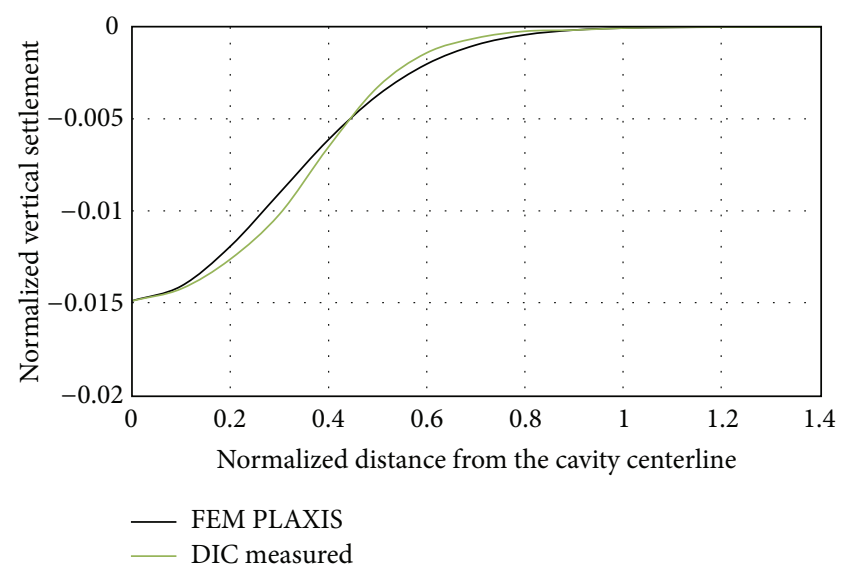

FIGURE 4: Transverse surface depression at pre-collapse.

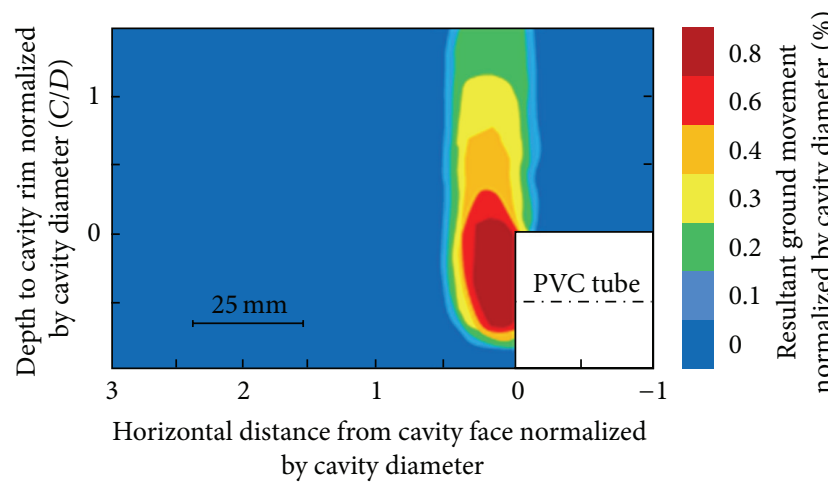

FIGURE 5: Contour plots of resultant ground movement normalized by cavity diameter.

4.2. Deformations at Precollapse Stage. Measured and computed transverse surface depression at precollapse, point $P_{f}$, is shown in Figure 4. The plot visualizes the extension of ground movement above the cavity in a plane perpendicular to the cylinder axis. The measured deformation was found to be consistent with the numerical modeling. A surface depression approximately equal to $1.5 \%$ of the cavity diameter $D$ was reported at the center of the cavity. The deformation extended horizontally to a distance equal to $1 \mathrm{D}$ from the cavity centerline.

Deformations in a plane parallel to the cylinder axis are presented in Figure 5 where contour plots of resultant ground movement normalized by cavity diameter are shown. It appears that deformations extend approximately $1 \mathrm{D}$ in front of the cavity. Unlike the deformations presented in Figure 4, these internal deformations (Figure 5) cannot be achieved by optical survey or surface monitoring instrumentation. The innovation of transparent soil allowed for measuring subsurface deformations in this experiment. Deformations were found to be much larger near the cavity and only minimally transferred to the surface. These findings highlight the importance of conducting a thorough impact assessment on nearby subsurface utilities and foundations for all subsurface construction projects. In practice, this issue seems to be overlooked with the application of grouting and other ground 


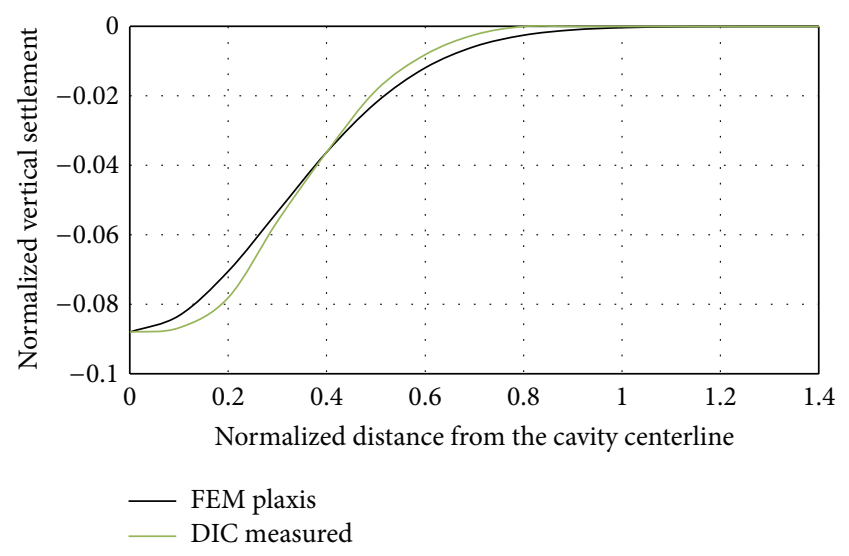

FIGURE 6: Transverse surface depression at collapsing stage.

improvement methods to control subsurface deformations. However, the quality of these underground techniques very much depends on the experience of the contractor and familiarity with the ground conditions which is questionable sometimes.

4.3. Postcollapse Deformations and Development of Sinkhole. After point $P_{f}$ was reached (Figure 3), further decrease of the supporting pressure led to sudden collapse (point $F$ ) as transparent soil tended to flow into the cavity and the failure envelope propagated progressively upward. The effect of these deformations on the surface was generating excessive surface disruption as shown in Figure 6. Again, the measured deformation was found to be consistent with the numerical modeling. A larger surface depression was reported at collapsing stage approximately. Surface depression at the center of the cavity was found to be equal to $9 \%$ of the cavity diameter $D$ and $8 \%$ larger than the values reported in Figure 4. Similar to precollapse, the deformation extended horizontally up to a distance equal to $1 \mathrm{D}$ from the cavity centerline. These findings indicate that the horizontal extension of deformation is independent of the magnitude of the cavity internal deformations. A similar conclusion was reported by Peck [15] and many other authors who studied settlement trough induced by tunneling.

$3 \mathrm{D}$ contour plots of resultant ground movements at collapsing stage are shown in Figure 7 for circular cavity of diameter $D$ with cover $C$ equal to $1.5 \mathrm{D}$. The figure visualizes the extension of ground movement above and ahead of the cavity. The dashed line in Figure 7 indicates the failed zone at the moment of collapse. Observed deformations were substantially larger than those observed at pre-collapse. Deformations were largest near the cavity but propagated upward to form a narrow chimney. The failed zone was relatively narrow and reached approximately $1 \mathrm{D}$ above the cavity crown, when arching of the soil prevented further deformation. The failed soil mass outcropped at the ground surface. The sinkhole appearance at the surface was found to be a small indicator of the final size of the deformations, as shown by the $3 \mathrm{D}$ plot (Figure 7). The failure of the overhanging soil around the cavity has led to a subsequent

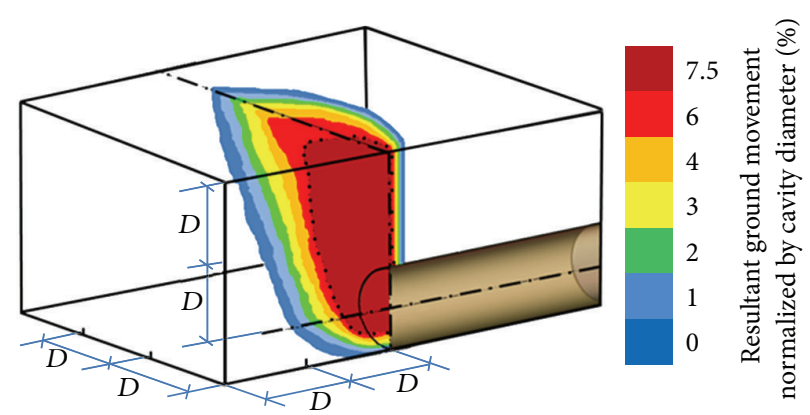

FIGURE 7: 3D deformations at collapsing stage.

funnel-shaped depression with a hole at the center. These results emphasize the need to extend the repaired zone following sinkhole formation by a minimum distance equal to $2 \mathrm{D}$ away and ahead of the cavity. Also some investigation should be conducted to evaluate the stability of buried utilities, foundation, and subgrade layers within this zone following sinkhole collapse.

\section{Conclusions}

Experimental technique and procedures were developed to analyze sinkhole pre-collapse and collapsing deformations. The use of transparent soil permitted visualization of actual internal ground deformations under the test conditions. Measured deformations parallel to the cavity axis were compared to numerical modeling and were found to be consistent. The observed failure mechanisms resembled a prismatic wedge in front of the cavity face extending upward in the form of a vertical chimney confirming the mechanisms proposed in the literature by various authors. The sinkhole appearance at the surface was found to be a small indicator of the final size and magnitude of the internal deformations as a subsequent funnel-shaped depression with a hole at the center developed. Therefore, the impact on nearby of utilities should be thought whenever underground construction is performed. Prediction of internal ground deformations is particularly important for ensuring stability of underground utilities and development of restoration plan after collapse occurred. Results of this study are believed to be of practical interest for predicting surface and internal ground deformations following sinkhole collapse. The modeling results emphasized the need to extend the repair zone following sinkhole collapse by a minimum distance that equals twice the cavity diameter away and ahead of the developed hole to cover pending failures illustrated by areas that experienced $1 \%$ to $6 \%$ deformations (resultant ground movement normalized by cavity diameter) as indicated in Figures 5 and 7 . These results can also be used to provide approximate bounds to areas affected by the sinkhole allowing for collapse risk to be assessed.

The good agreement between transparent soil and numerical modeling confirms that transparent soil is a valid tool for studying sinkhole collapse. The results presented here 
can be used to provide models for predicting soil behavior around collapsing cavities. It can also assist the transportation agencies and contractors to predict unseen or buried unstable areas of a roadway after sinkhole collapse and develop a comprehensive restoration plan.

It is important to mention that these results were controlled by specific tests conditions (i.e., geotechnical properties, cavity depth, diameter, surcharge pressure, surface pressure, and boundary conditions). Further studies continuing from this research could look at different ground conditions, such as different densities, layered soil, and interaction with nearby utilities and foundations.

\section{Acknowledgments}

Research on transparent soils is currently supported by the Defense Threat Reduction Agency under Grant no. HDTRA110-1-0049. Transparent soils were originally developed with the National Science Foundation funding under Career Grant no. CMS9733064. Continued NSF funding under Grants nos. DGE741714 and DGE0337668 is gratefully acknowledged.

\section{References}

[1] W. H. Craig, "Collapse of cohesive overburden following removal of support," Canadian Geotechnical Journal, vol. 27, no. 3, pp. 355-364, 1990.

[2] W. A. Abdulla and D. J. Goodings, "Modeling of sinkholes in weakly cemented sand," Journal of Geotechnical and Geoenvironmental Engineering, vol. 122, no. 12, pp. 998-1005, 1996.

[3] J. H. Atkinson and D. M. Potts, "Subsidence above Shallow circular tunnels in soft ground," Journal of Geotechnical Engineering Division, vol. 103, no. 4, pp. 307-325, 1977.

[4] E. H. Davis, M. J. Gunn, R. J. Mair, and H. N. Seneviratne, "The stability of shallow tunnels and underground openings in cohesive material," Geotechnique, vol. 30, no. 4, pp. 397-416, 1980.

[5] H.-B. Mühlhaus, "Lower bound solutions for circular tunnels in two and three dimensions," Rock Mechanics and Rock Engineering, vol. 18, no. 1, pp. 37-52, 1985.

[6] E. Leca and L. Dormieux, "Upper and lower bound solutions for the face stability of shallow circular tunnels in frictional material," Geotechnique, vol. 40, no. 4, pp. 581-606, 1990.

[7] M. Ahmed and M. Iskander, "Analysis of tunneling-induced ground movements using transparent soil models," Journal of Geotechnical and Geoenvironmental Engineering, vol. 137, no. 5, pp. 525-535, 2011.

[8] M. Ahmed and M. Iskander, "Evaluation of tunnel face stability by transparent soil models," Tunnelling and Underground Space Technology, vol. 27, no. 1, pp. 101-110, 2012.

[9] M. Iskander, Modelling With Transparent Soils, Visualizing Soil Structure Interaction and Multi Phase Flow, Non-Intrusively, Springer, Dordrecht, The Netherlands, 2010.

[10] S. Sadek, M. G. Iskander, and J. Liu, "Geotechnical properties of transparent silica," Canadian Geotechnical Journal, vol. 39, no. 1, pp. 111-124, 2002.

[11] R. Taylor, R. Grant, S. Robson, and J. Kuwano, "An image analysis system for determining plane and 3D displacements in soil models," in Proceedings of Centrifuge '89, pp. 73-78, Taylor and Francis, London, UK, 1998.
[12] S. G. Paikowsky and F. Xi, "Particle motion tracking utilizing a high-resolution digital CCD camera," Geotechnical Testing Journal, vol. 23, no. 1, pp. 123-134, 2000.

[13] D. R. Gill and B. M. Lehane, "An optical technique for investigating soil displacement patterns," Geotechnical Testing Journal, vol. 24, no. 3, pp. 324-329, 2001.

[14] Dantec Dynamics, Flow Manager Software User Guide, Tonsbakken, Denmark, 2001.

[15] R. B. Peck, "Deep excavations and tunneling in soft ground," in Proceedings of the 7th International Conference on Soil Mechanics and Foundation Engineering, Mexico City: State ofthe-Art Volume, pp. 225-290, 1969. 

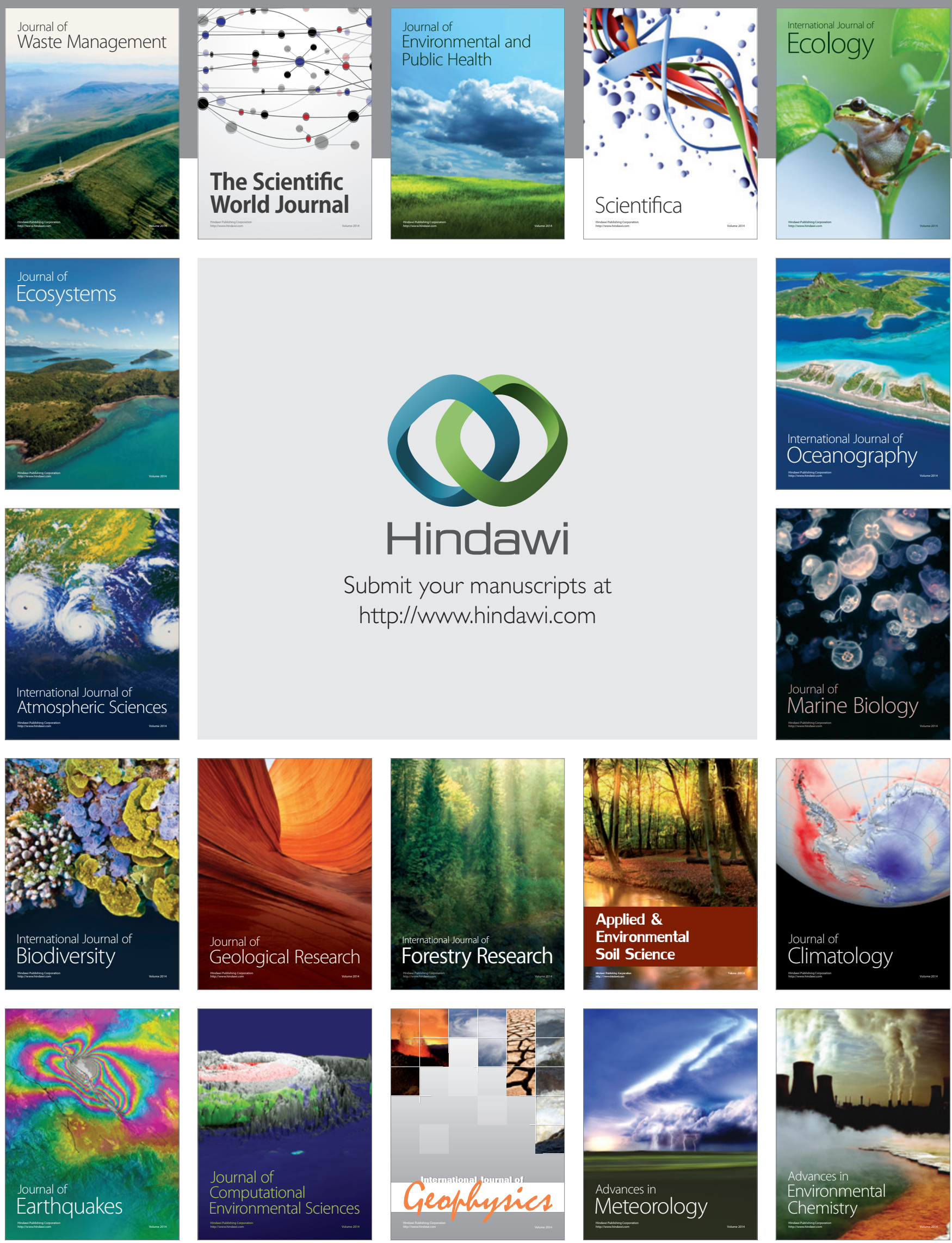